\title{
Solutions of the boundary value problem for a $2 n$ th-order nonlinear difference equation containing both advance and retardation
}

Qian Wang ${ }^{1,2}$ and Zhan Zhou ${ }^{1,2^{*}}$

\section{*Correspondence:}

zzhou0321@hotmail.com

'School of Mathematics and

Information Science, Guangzhou

University, Guangzhou, Guangdong 510006, P.R. China

${ }^{2}$ Key Laboratory of Mathematics and Interdisciplinary Sciences of

Guangdong Higher Education Institutes, Guangzhou University,

Guangzhou, 510006, P.R. China

\begin{abstract}
In this paper, we consider the boundary value problem for a 2nth-order nonlinear difference equation containing both advance and retardation. By using the critical point theory, some sufficient conditions of the existence of solutions of the boundary value problem are obtained. The proof is based on the linking theorem. An example is given to illustrate our results.
\end{abstract}

Keywords: boundary value problem; difference equation; linking theorem; 2nth-order

\section{Introduction}

Consider the following $2 n$ th-order nonlinear difference equation:

$$
\Delta^{n}\left(r_{t-n} \Delta^{n} x_{t-n}\right)+(-1)^{n+1} f\left(t, x_{t+1}, x_{t}, x_{t-1}\right)=0, \quad t=1,2, \ldots, T,
$$

with boundary value conditions

$$
x_{j}=0, \quad j=1-n, 2-n, \ldots, 0, T+1, \ldots, T+n,
$$

where $T$ and $n$ are given positive integers with $T>n, f(t, \cdot): \mathbb{R}^{3} \rightarrow \mathbb{R}$ is continuous for $t=1,2, \ldots, T, r_{1-n}, r_{2-n}, \ldots, r_{T}$ are nonzero real numbers and $\Delta$ is the forward difference operator defined by $\Delta x_{n}=x_{n+1}-x_{n}, \Delta^{2} x_{n}=\Delta\left(\Delta x_{n}\right)$.

In the last decade, by using various techniques such as critical point theory, fixed point theory, topological degree theory and coincidence degree theory, a great deal of works have been done on the existence of solutions to boundary value problems of difference equations (see [1-7] and references therein). Among these approaches, the critical point theory seems to be a powerful tool to solving this problem (see [5, 7-9]). However, compared to the boundary value problems of lower order difference equations $[6,8,10-$ 13], the study of boundary value problems of higher order difference equations is relatively less (see $[9,14,15])$, especially the works done by using the critical point theory. Therefore, there is still spacious room to explore the boundary value problems of higher-order difference equations. For the background on difference equations, we refer to [16]. 
Recently, in [17], the author considered the $2 n$ th-order nonlinear difference equation

$$
\Delta^{n}\left(r_{t-n} \Delta^{n} x_{t-n}\right)+(-1)^{n+1} f\left(t, x_{t}\right)=0, \quad t=1,2, \ldots, T
$$

with the boundary value conditions (1.2). He distinguished two cases that $f(t, z)$ is superlinear and sublinear in the second variable $z$, respectively. Equation (1.3) represents a class of no-delay difference equations.

In this paper, we consider the existence of solutions of the boundary value problem of (1.1) with (1.2). First, we construct a functional $J$ such that solutions of boundary value problem (1.1) with (1.2) correspond to critical points of $J$. Then, by using the linking theorem, we obtain the existence of critical points of $J$. Finally, an example is given to illustrate our results. We mention that (1.1) is a kind of difference equation containing both advance and retardation. This kind of difference equation has many applications both in theory and practice. For example, as a special case of $n=1,(1.1)$ may be thought to be the discrete analogue of the following forward and backward differential difference equation:

$$
c^{2} u^{\prime \prime}(t)=V^{\prime}(u(t+1)-u(t))-V^{\prime}(u(t)-u(t-1)), \quad t \in \mathbb{R} .
$$

Equation (1.4) has been studied extensively by many scholars. For example, Smets and Willem [18] obtained the existence of solitary waves of (1.4).

In [7], the authors considered (1.1) for the case when $n=1$ with different boundary value conditions

$$
\Delta x_{0}=A, \quad x_{T+1}=B .
$$

In [16], Agarwal took the following difference equation:

$$
-\omega^{2} M u(k)+f(-u(k-1)+2 u(k)-u(k+1))=0
$$

with the boundary value conditions

$$
u(0)=u(k+1)=0
$$

as an example. It represents the amplitude of the motion of every particle in the string.

Throughout this paper, we assume that there exists a function $F(t, x, y)$ which is differentiable in variable $x$ and $y$ defined for $t=0,1, \ldots, T$, satisfies

$$
\frac{\partial F(t-1, y, z)}{\partial y}+\frac{\partial F(t, x, y)}{\partial y}=f(t, x, y, z), \quad \forall x, y, z \in \mathbb{R}
$$

for $t=1,2, \ldots, T$.

\section{Preliminaries and main results}

Before we apply the critical point theory, we shall establish the corresponding variational framework for (1.1) with (1.2).

Let $\mathbb{N}, \mathbb{Z}$ and $\mathbb{R}$ denote the sets of all natural numbers, integers and real numbers, respectively. For $a, b \in \mathbb{Z}$, define $\mathbb{Z}(a)=\{a, a+1, \ldots\}, \mathbb{Z}(a, b)=\{a, a+1, \ldots, b\}$ when $a \leq b$. 
Let

$$
E=\left\{x=\left\{x_{t}\right\}: \mathbb{Z}(1-n, T+n) \rightarrow \mathbb{R} \mid x_{1-i}=x_{T+i}=0, i \in \mathbb{Z}(1, n)\right\} .
$$

Then $E$ is a $T$-dimensional Hilbert space.

Obviously, $E$ is isomorphic to $\mathbb{R}^{T}$. In fact, we can find a map $I: E \rightarrow \mathbb{R}^{T}$ defined by

$$
I:\left\{x_{t}\right\} \rightarrow\left(x_{1}, x_{2}, \ldots, x_{T}\right)^{\mathrm{tr}},
$$

where ${ }^{\text {tr }}$ means the transpose of $\cdot$.

Define the inner product on $E$ as

$$
\langle x, y\rangle=\sum_{i=1}^{T} x_{i} y_{i}, \quad \forall x, y \in E
$$

The corresponding norm $\|\cdot\|$ can be induced by

$$
\|x\|=\left(\sum_{t=1}^{T}\left|x_{t}\right|^{2}\right)^{\frac{1}{2}}, \quad \forall x \in E .
$$

For all $x \in E$, define the functional $J(x)$ on $E$ as follows:

$$
J(x)=\frac{1}{2} \sum_{t=1-n}^{T} r_{t}\left(\Delta^{n} x_{t}\right)^{2}-\sum_{t=0}^{T} F\left(t, x_{t+1}, x_{t}\right) .
$$

Clearly, $J(x) \in C^{1}(E, \mathbb{R})$.

By using the fact that

$$
\Delta^{n} x_{t-n}=\sum_{i=0}^{n}(-1)^{n-i}\left(\begin{array}{c}
n \\
i
\end{array}\right) x_{t-n+i}
$$

we can compute the partial derivative as

$$
\frac{\partial J(x)}{\partial x_{t}}=(-1)^{n} \Delta^{n}\left(r_{t-n} \Delta^{n} x_{t-n}\right)-f\left(t, x_{t+1}, x_{t}, x_{t-1}\right)
$$

for $t \in \mathbb{Z}(1, T)$. Therefore, $x \in E$ is a critical point of $J$ if and only if $x$ is a solution of (1.1) with (1.2).

By a simple computation, we see that there exists a $T \times T$ matrix $A$ such that

$$
\sum_{t=1-n}^{T} r_{t}\left(\Delta^{n} x_{t}\right)^{2}=(A I(x), I(x))
$$

for all $x \in E$. Therefore, we can write $J(x)$ as

$$
J(x)=\frac{1}{2}(A I(x), I(x))-F(x),
$$

where $F(x)=\sum_{t=0}^{T} F\left(t, x_{t+1}, x_{t}\right)$. 
By a straightforward calculation, we obtain that

$$
\operatorname{tr} A=\sum_{i=1-n}^{0} r_{i} \sum_{j=0}^{i+n-1}\left(\begin{array}{l}
n \\
j
\end{array}\right)^{2}+\sum_{i=1}^{T-n} r_{i} \sum_{j=0}^{T-n}\left(\begin{array}{l}
n \\
j
\end{array}\right)^{2}+\sum_{i=T-n+1}^{T} r_{i} \sum_{j=0}^{T-i}\left(\begin{array}{l}
n \\
j
\end{array}\right)^{2} .
$$

Let $\lambda(A), \lambda^{+}(A)$ and $\lambda^{-}(A)$ be the sets of all the eigenvalues of $A$, all the positive eigenvalues of $A$ and all the negative eigenvalues of $A$, respectively. Define $\lambda_{\max }=\max \{\lambda: \lambda \in \lambda(A)\}$, $\lambda_{\min }=\min \{\lambda: \lambda \in \lambda(A)\}$. If $\lambda^{+}(A) \neq \phi$, let $\lambda_{\max }^{+}=\max \left\{\lambda: \lambda \in \lambda^{+}(A)\right\}, \lambda_{\min }^{+}=\min \{\lambda: \lambda \in$ $\lambda^{+}(A)$ \}. Let $A^{0}, A^{+}$and $A^{-}$denote the eigenspaces associated with the 0 eigenvalue, all positive eigenvalues and all negative eigenvalues of $A$, respectively. It follows that

$$
\frac{1}{2} \lambda_{\min }\|x\|^{2}-F(x) \leq J(x) \leq \frac{1}{2} \lambda_{\max }\|x\|^{2}-F(x)
$$

and

$$
\mathbb{R}^{T}=A^{0} \oplus A^{+} \oplus A^{-}
$$

Definition 2.1 Let $E$ be a real Banach space, the functional $J \in C^{1}(E, \mathbb{R})$ is said to satisfy the Palais-Smale (P.S. for short) condition if any sequence $\left\{x_{m}\right\}$ in $E$, such that $\left\{J\left(x_{m}\right)\right\}$ is bounded and $J^{\prime}\left(x_{m}\right) \rightarrow 0$ as $m \rightarrow \infty$, contains a convergent subsequence.

Let $B_{\rho}$ denote the open ball in $E$ with radius $\rho$ and center 0 , and let $\partial B_{\rho}$ denote its boundary.

In order to obtain the existence of critical points of $J$ on $E$, we cite the basic lemma, which is crucial in the proof of our main results.

Lemma 2.1 (Linking theorem [19]) Let $E$ be a real Hilbert space and $E=E_{1} \oplus E_{2}$, where $E_{1}$ is a finite-dimensional subspace of $E$. Assume that $J \in C^{1}(E, \mathbb{R})$ satisfies the P.S. condition and the following two conditions.

$\left(\mathrm{J}_{1}\right)$ There exist constants $a>0$ and $\rho>0$ such that $\left.J\right|_{\partial B_{\rho} \cap E_{2}} \geq a$;

$\left(\mathrm{J}_{2}\right)$ There exist an $e \in \partial B_{1} \cap E_{2}$ and a constant $R_{0}>\rho$ such that $\left.J\right|_{\partial Q} \leq 0$, where $Q \triangleq\left(B_{R_{0}} \cap\right.$ $\left.E_{1}\right) \oplus\left\{r e \mid 0<r<R_{0}\right\}$.

Then J possesses a critical value $c \geq a$. Moreover, $c$ can be characterized as

$$
c=\inf _{h \in \Gamma} \max _{x \in \bar{Q}} J(h(x))
$$

where $\Gamma=\left\{h \in C(\bar{Q}, E):\left.h\right|_{\partial Q}=\operatorname{id}_{\partial Q}\right\}$ and $\operatorname{id}_{\partial Q}$ is the identity operator on $\partial Q$.

Now we state our main results.

Theorem 2.1 Assume that the following conditions are satisfied:

(A1)

$$
\sum_{i=1-n}^{0} r_{i} \sum_{j=0}^{i+n-1}\left(\begin{array}{l}
n \\
j
\end{array}\right)^{2}+\sum_{i=1}^{T-n} r_{i} \sum_{j=0}^{T-n}\left(\begin{array}{l}
n \\
j
\end{array}\right)^{2}+\sum_{i=T-n+1}^{T} r_{i} \sum_{j=0}^{T-i}\left(\begin{array}{l}
n \\
j
\end{array}\right)^{2}>0 .
$$


(A2)

$$
\liminf _{x^{2}+y^{2} \rightarrow \infty} \frac{F(t, x, y)}{x^{2}+y^{2}}>\frac{1}{4} \lambda_{\max }, \quad t \in \mathbb{Z}(0, T) .
$$

(A3)

$$
\lim _{(x, y) \rightarrow(0,0)} \frac{F(t, x, y)}{x^{2}+y^{2}}=0, \quad t \in \mathbb{Z}(0, T) .
$$

Then (1.1) with (1.2) possesses at least two nontrivial solutions.

Corollary 2.1 Assume that (A1) holds, and $F(t, x, y)$ satisfies (A3) and

(A4) There exist constants $R>0$ and $\beta>2$ such that

$$
\begin{aligned}
0 & <\beta F(t, x, y) \\
& \leq \frac{\partial F(t, x, y)}{\partial x} x+\frac{\partial F(t, x, y)}{\partial y} y, \quad \forall \sqrt{x^{2}+y^{2}} \geq R \text { and } t \in \mathbb{Z}(0, T) .
\end{aligned}
$$

Then (1.1) with (1.2) possesses at least two nontrivial solutions.

Remark 2.1 If $r_{i}>0$ for $i \in \mathbb{Z}(1-n, T)$, then (2.12) clearly holds.

Remark 2.2 Assumption (A4) is a condition similar to the classical AmbrosettiRabinowitz superlinear condition [19], which implies that there exist constants $a_{1}>0$ and $a_{2}>0$ such that

$$
F(t, x, y) \geq a_{1}\left(\sqrt{x^{2}+y^{2}}\right)^{\beta}-a_{2}, \quad \forall(t, x, y) \in \mathbb{Z}(0, T) \times \mathbb{R}^{2},
$$

which leads to

$$
\min _{t \in \mathbb{Z}(0, T)} \liminf _{x^{2}+y^{2} \rightarrow \infty} \frac{F(t, x, y)}{x^{2}+y^{2}}=+\infty
$$

and (A2) is satisfied. So Corollary 2.1 holds.

\section{Proof of Theorem 2.1}

Lemma 3.1 Assume that (A2) holds, then the functional J satisfies the P.S. condition.

Proof According to (A2), there exist positive constants $a_{3}>\lambda_{\max } / 4$ and $a_{4}$ such that

$$
F(t, x, y) \geq a_{3}\left(x^{2}+y^{2}\right)-a_{4}, \quad \forall(t, x, y) \in \mathbb{Z}(0, T) \times \mathbb{R}^{2} .
$$

Let $\left\{x^{(k)}\right\}$ be a sequence in $E$ such that $\left\{J\left(x^{(k)}\right)\right\}$ is bounded and $J^{\prime}\left(x^{(k)}\right) \rightarrow 0$ as $k \rightarrow \infty$. Then there exists a positive constant $C$ such that $\left|J\left(x^{(k)}\right)\right| \leq C$. 
By (2.8) and (2.10), we have

$$
\begin{aligned}
J\left(x^{(k)}\right) & =\frac{1}{2}\left(A x^{(k)}, x^{(k)}\right)-F\left(x^{(k)}\right) \\
& \leq \frac{1}{2} \lambda_{\max }\left\|x^{(k)}\right\|^{2}-a_{3} \sum_{t=0}^{T}\left(\left(x_{t}^{(k)}\right)^{2}+\left(x_{t+1}^{(k)}\right)^{2}\right)+(T+1) a_{4} \\
& \leq \frac{1}{2}\left(\lambda_{\max }-4 a_{3}\right)\left\|x^{(k)}\right\|^{2}+(T+1) a_{4} .
\end{aligned}
$$

On the other hand, $J\left(x^{(k)}\right) \geq-C$. Combining this with (3.2), we have

$$
\left\|x^{(k)}\right\|^{2} \leq \frac{2(T+1) a_{4}+2 C}{4 a_{3}-\lambda_{\max }}
$$

Since $E$ is a finite-dimensional space, (3.3) implies $\left\{x^{(k)}\right\}$ has a convergent subsequence. Thus P.S. condition is verified.

Now we give the proof of Theorem 2.1.

Proof According to (A3), it is easy to see that $F(t, 0,0)=0$. Therefore, $\{0\}$ is a solution of (1.1) with (1.2) which is called a trivial solution. From (A1), $\operatorname{tr}(A)>0$, then we conclude that matrix $A$ has at least one positive eigenvalue and $\lambda_{\max }=\lambda_{\max }^{+}$. By (3.2), we have

$$
J(x) \leq \frac{1}{2}\left(\lambda_{\max }^{+}-4 a_{3}\right)\|x\|^{2}+(T+1) a_{4}
$$

This implies that $J(x) \rightarrow-\infty$ as $\|x\| \rightarrow \infty$, then $-J(x)$ is a coercive map. It implies that $J$ is bounded above on $E$. By the continuity of $J$ on $E$, there exists $m \in E$ such that

$$
J(m)=\max \{J(x) \mid x \in E\}=\bar{c} .
$$

Clearly, $m$ is a critical point of $J$ on $E$. We prove that $m$ is nontrivial.

In fact, from (A3), there exists $\rho_{0}>0$ such that, for any $(x, y) \in \mathbb{R}^{2}$ with $x^{2}+y^{2} \leq \rho_{0}^{2}$ and $t \in \mathbb{Z}(0, T)$, we have

$$
F(t, x, y) \leq \frac{1}{8} \lambda_{\min }^{+}\left(x^{2}+y^{2}\right)
$$

For any $x \in E$ with $I(x) \in A^{+}$and $\|x\|=\rho_{0}$, by (2.10), it is clear that

$$
\begin{aligned}
J(x) & \geq \frac{1}{2} \lambda_{\min }^{+}\|x\|^{2}-\frac{1}{8} \lambda_{\min }^{+} \sum_{t=0}^{T}\left(x_{t}^{2}+x_{t+1}^{2}\right) \\
& =\frac{1}{2} \lambda_{\min }^{+}\|x\|^{2}-\frac{1}{4} \lambda_{\min }^{+}\|x\|^{2} \\
& =\frac{1}{4} \lambda_{\min }^{+}\|x\|^{2} \\
& =\frac{1}{4} \lambda_{\min }^{+} \rho_{0}^{2} .
\end{aligned}
$$


Therefore, $\bar{c}=\max \{J(x) \mid x \in E\} \geq \frac{1}{4} \lambda_{\min }^{+} \rho_{0}^{2}>0$, which implies that $m \neq 0$. That is, $m$ is a nontrivial critical point of $J$.

Let $E_{2}=I^{-1}\left(A^{+}\right), E_{1}=I^{-1}\left(A^{-} \oplus A^{0}\right)$ and $a=\frac{1}{4} \lambda_{\min } \rho_{0}^{2}>0$, then we have

$$
E=E_{1} \oplus E_{2}
$$

and

$$
\left.J(x)\right|_{\partial B_{\rho_{0}} \cap E_{2}} \geq a .
$$

Let $e \in \partial B_{1} \cap E_{2}$, for any $z \in E_{1}$ and $r \in \mathbb{R}$, we have

$$
\begin{aligned}
J(r e+z) & =\frac{1}{2}(A I(r e+z), I(r e+z))-F(r e+z) \\
& \leq \frac{1}{2} r^{2}(A I(e), I(e))+\frac{1}{2}(A I(z), I(z))-2 a_{3}\|r e+z\|^{2}+(T+1) a_{4} \\
& \leq \frac{1}{2} \lambda_{\max }^{+} r^{2}-2 a_{3}\left(r^{2}+\|z\|^{2}\right)+(T+1) a_{4} \\
& =\frac{1}{2}\left(\lambda_{\max }^{+}-4 a_{3}\right) r^{2}-2 a_{3}\|z\|^{2}+(T+1) a_{4} .
\end{aligned}
$$

Since $J(z) \leq 0$ for $z \in E_{1}$, we see that there exists a positive constant $R_{0}>\rho_{0}$ such that $J(x) \leq 0$ for any $x \in \partial Q$, where $Q \triangleq\left(B_{R_{0}} \cap E_{1}\right) \oplus\left\{r e \mid 0<r<R_{0}\right\}$. By the linking theorem, $J$ possesses a critical value $\tilde{c} \geq a$, where

$$
\tilde{c}=\inf _{h \in \Gamma} \max _{x \in \bar{Q}} J(h(x)) \quad \text { and } \quad \Gamma=\left\{h \in C\left(\bar{Q}, \mathbb{R}^{T}\right):\left.h\right|_{\partial Q}=\operatorname{id}_{\partial Q}\right\} .
$$

For convenience, we note $J\left(m^{*}\right)=\tilde{c}, m^{*} \in E$. Obviously, $m^{*} \neq\{0\}$.

If $m \neq m^{*}$, then the proof is finished. Otherwise, $\bar{c}=J(m)=J\left(m^{*}\right)=\tilde{c}$. Since $e$ is arbitrary, we can choose $-e \in \partial B_{1} \cap E_{2}$. Similarly, there exists $R_{*}>\rho_{0}$ such that $J(x) \leq 0$ for any $x \in$ $\partial Q_{1}$, where $Q_{1} \triangleq\left(B_{R_{*}} \cap E_{1}\right) \oplus\left\{-r e \mid 0<r<R_{*}\right\}$. Again, by the linking theorem, $J$ possesses a critical value $\hat{c} \geq a$, where

$$
\hat{c}=\inf _{h \in \Gamma_{1}} \max _{x \in \bar{Q}_{1}} J(h(x)) \quad \text { and } \quad \Gamma_{1}=\left\{h \in C\left(\bar{Q}_{1}, \mathbb{R}^{T}\right):\left.h\right|_{\partial Q_{1}}=\operatorname{id}_{\partial Q_{1}}\right\} .
$$

Note $J(\hat{m})=\hat{c}$. We will assert that $m^{*} \neq \hat{m}$.

By way of contradiction, assume that $m^{*}=\hat{m}$. Then $\hat{c}=\tilde{c}=\bar{c}=\max \{J(x) \mid x \in E\}$. Since $\left.J(x)\right|_{\partial Q} \leq 0$ and $\left.J(x)\right|_{\partial Q_{1}} \leq 0, \hat{m}$ must be an interior point of both sets $Q$ and $Q_{1}$. However, $Q \cap Q_{1} \subset E_{1}$ and $J(x) \leq 0$ for any $x \in E_{1}$. This implies that $\bar{c}<0$. It is a contradiction, so the conclusion of Theorem 2.1 holds.

Now the proof is complete.

\section{Example}

As an application of Theorem 2.1, we give an example to illustrate our result.

Example 4.1 Consider the following $2 n$ th-order difference equation:

$$
\Delta^{n}\left(r_{t-n} \Delta^{n} x_{t-n}\right)+(-1)^{n+1} f\left(t, x_{t+1}, x_{t}, x_{t-1}\right)=0, \quad t \in \mathbb{Z}(1, T)
$$


with boundary value conditions

$$
x_{j}=0, \quad j \in \mathbb{Z}(1-n, 0) \cup \mathbb{Z}(T+1, T+n),
$$

where $T$ and $n$ are given positive integers with $T>n, r_{t}=-1$ for $t \in \mathbb{Z}(1-n, 0), r_{t}=2$ for $t \in \mathbb{Z}(1, T)$, and

$$
\begin{aligned}
f(t, x, y, z)= & 2 y\left(2+\sin \frac{t \pi}{T}\right)\left(\frac{x^{2}+y^{2}}{2+y^{2}} \ln \left(2+x^{2}\right)+\ln \left(2+x^{2}\right) \ln \left(2+y^{2}\right)-\ln ^{2} 2\right) \\
& +2 y\left(2+\sin \frac{(t-1) \pi}{T}\right)\left(\frac{y^{2}+z^{2}}{2+y^{2}} \ln \left(2+z^{2}\right)+\ln \left(2+y^{2}\right) \ln \left(2+z^{2}\right)-\ln ^{2} 2\right)
\end{aligned}
$$

for $t \in \mathbb{Z}(1, T)$. Clearly, (2.12) holds. If we let

$$
F(t, x, y)=\left(2+\sin \frac{t \pi}{T}\right)\left(\ln \left(2+x^{2}\right) \ln \left(2+y^{2}\right)-\ln ^{2} 2\right)\left(x^{2}+y^{2}\right), \quad t \in \mathbb{Z}(0, T),
$$

then (1.8) is satisfied. Since

$$
\lim _{x^{2}+y^{2} \rightarrow \infty} \frac{F(t, x, y)}{x^{2}+y^{2}}=+\infty, \quad \lim _{x^{2}+y^{2} \rightarrow 0} \frac{F(t, x, y)}{x^{2}+y^{2}}=0
$$

for $t \in \mathbb{Z}(0, T)$, we see that (2.13) and (2.14) hold. By Theorem 2.1, we know that (4.1) with (4.2) has at least two nontrivial solutions.

\section{Competing interests}

The authors declare that they have no competing interests.

\section{Authors' contributions}

All authors jointly worked on the results, and they read and approved the final manuscript.

\section{Acknowledgements}

The authors would like to thank the anonymous referee for his/her valuable suggestions. This work is supported by Program for Changjiang Scholars and Innovative Research Team in University (No. IRT1226), the Specialized Fund for the Doctoral Program of Higher Education of China (No. 20114410110002), and SRF of Guangzhou Education Bureau (No. 10A012)

Received: 22 August 2013 Accepted: 18 October 2013 Published: 18 Nov 2013

\section{References}

1. Henderson, J, Thompson, H: Existence of multiple solutions for second-order discrete boundary value problems. Comput. Math. Appl. 43, 1239-1248 (2002)

2. Li, YK, Shu, JY: Solvability of boundary value problems with Riemann-Stieltjes $\Delta$-integral conditions for second-order dynamic equations on time scales at resonance. Adv. Differ. Equ. 2011(42), 1-18 (2011)

3. Ma, RY, Gao, CH: Bifurcation of positive solutions of a nonlinear discrete fourth-order boundary value problem. Z. Angew. Math. Phys. 64, 493-506 (2013)

4. Yuan, CJ: Positive solutions of a singular positone and semipositone boundary value problems for fourth-order difference equations. Discrete Dyn. Nat. Soc. 2010, Article ID 312864 (2010)

5. Gao, LM: Existence of multiple solutions for a second-order difference equation with a parameter. Appl. Math. Comput. 216, 1592-1598 (2010)

6. Liang, HH, Weng, PX: Existence and multiple solutions for a second-order difference boundary value problem via critical point theory. J. Math. Anal. Appl. 326, 511-520 (2007)

7. Deng, XQ, Shi, HP: On boundary value problems for second order nonlinear functional difference equations. Acta Appl. Math. 110, 1277-1287 (2010)

8. Liu, JS, Wang, SL, Zhang, JM: Multiple solutions for boundary value problems of second-order difference equations with resonance. J. Math. Anal. Appl. 374, 187-196 (2011)

9. Zou, QR, Weng, PX: Solutions of $2 n$ th-order boundary value problem for difference equation via variational method. Adv. Differ. Equ. 2009, Article ID 730484 (2009) 
10. Bai, DY, Xu, YT: Nontrivial solutions of boundary value problems of second-order difference equations. J. Math. Anal. Appl. 326, 297-302 (2007)

11. Zhang, R: Positive solutions of BVPs for third-order discrete nonlinear difference systems. J. Appl. Math. Comput. 35, 551-575 (2011)

12. Yu, JS, Guo, ZM: Boundary value problems of discrete generalized Emden-Fowler equation. Sci. China Ser. A 49 1303-1314 (2006)

13. He, TS, Xu, YT: Positive solutions for nonlinear discrete second-order boundary value problems with parameter dependence. J. Math. Anal. Appl. 379, 627-636 (2011)

14. Berger, $\mathrm{H}$ : Existence of nontrivial solutions of a two-point boundary value problem for a $2 n$ th-order nonlinear difference equation. Adv. Dyn. Syst. Appl. 3, 131-146 (2008)

15. Xie, SL, Zhu, J: Positive solutions of the system for $n$ th-order singular nonlocal boundary value problems. J. Appl. Math. Comput. 37, 119-132 (2011)

16. Agarwal, RP: Difference Equations and Inequalities: Theory, Methods and Applications, 2nd edn. Marcel Dekker., New York (2000)

17. Zhou, Z: Boundary value problems for a class of higher order nonlinear difference equations. J. Guangzhou Univ. (Nat. Sci. Ed.) 11, 1-4 (2012) (in Chinese)

18. Smets, D, Willem, M: Solitary waves with prescribed speed on infinite lattices. J. Funct. Anal. 149, $266-275$ (1997)

19. Rabinowitz, PH: Minimax Methods in Critical Point Theory with Applications to Differential Equations, vol. 65. Am. Math. Soc., Providence (1986)

10.1186/1687-1847-2013-322

Cite this article as: Wang and Zhou: Solutions of the boundary value problem for a 2 nth-order nonlinear difference equation containing both advance and retardation. Advances in Difference Equations 2013, 2013:322

\section{Submit your manuscript to a SpringerOpen ${ }^{\circ}$ journal and benefit from:}

- Convenient online submission

- Rigorous peer review

- Immediate publication on acceptance

Open access: articles freely available online

- High visibility within the field

- Retaining the copyright to your article 